\title{
Advanced Electron Microscopy for Energy Related Materials
}

\author{
Ryo Ishikawa $^{1}$, Naoya Shibata ${ }^{1,2}$ and Yuichi Ikuhara ${ }^{1,3,4}$ \\ 1. Institute of Engineering Innovation, University of Tokyo, Bunkyo, Tokyo, 113-8656, Japan. \\ 2. Japan Science and Technology Agency, PRESTO, Saitama 332-0012, Japan \\ 3. WPI Research Center, AIMR, Tohoku University, Sendai 980-8577, Japan \\ 4. Nanostructures Research Laboratory, Japan Fine Ceramics Center, Atsuta, Nagoya 456-8587, Japan
}

Recent progress on electron optics promotes the capability of scanning transmission electron microscopy (STEM) and it becomes versatile tool for solving materials problems at atomic scale. The most popular ADF imaging mode has a strong sensitivity of the atomic number (Z-contrast) and one can see even single heavy dopants, but it is insensitive to the light elements. On the other hand, recently developed annular bright-field (ABF) imaging is capable to directly see oxygen, lithium and even hydrogen atoms within thin crystals $[1,2]$, and now ABF imaging is routinely used for the determination of atomic structures of energy related materials. Fig. 1 shows the typical ADF and ABF STEM images obtained from $\mathrm{Li}_{2} \mathrm{TMO}_{3}$ in which oxygen and lithium atoms are basically invisible in ADF but can see these atoms in ABF image. In this presentation, we show the basic image formation of ABF STEM and some applications of $\mathrm{ABF} / \mathrm{ADF}$ for light element materials such as lithium ion battery [3] or photocatalytic materials [4].

Manganese-rich lithium oxide $\left(\mathrm{Li}_{1+x} \mathrm{TM}_{1-x} \mathrm{O}_{2}\right.$, TM: transition metal $)$ has actively investigated as cathode materials of lithium ion battery. On the basis of X-ray or neutron diffraction, it was revealed that the material consists of two phases such as rhombohedral and monoclinic structures, but the spatial distribution of two phases is still under controversial. The typical grain size is a few micrometers and we often found two phases in single grains through conventional electron diffraction, suggesting that the size of two phases (or domains) is relatively small. So it requires atomic-resolution observation to identify whether interlayer growth or two-phase separation. In the bulk regions of $\mathrm{Li}_{2} \mathrm{TMO}_{3}$, it is found several stacking faults along $c$-axis or a few layer inter growth of monoclinic-type structures, which gives not new spots but diffuse streaks in diffraction pattern. After the heavy investigation, we found two-phase separation and it forms hetero-interface along $c$-axis, which is directly observed by ABF/ADF STEM imaging.

The other example is photocatalytic water splitting oxynitride of $\mathrm{LaTiO}_{2} \mathrm{~N}$. The photocatalytic reaction occurs on the surface and hence it is important to understand the surface atomic structure. Fig. 2(a) shows ABF STEM image obtained from the surface atomic structure of $\mathrm{LaTiO}_{2} \mathrm{~N}$ after annealing in the air. One can see the reconstructed atomic structure on the first top-surface layer. When the specimen is quickly treated with aqua regia ( 15 seconds), it removes only surface reconstructed regions. This quick treatment significantly improves the photocatalytic activity for both the $\mathrm{H}_{2}$ and $\mathrm{O}_{2}$ evolution reaction, which is firstly realized by ABF/ADF STEM imaging. Details and further examples will be shown in this presentation. 


\section{References}

[1] SD Findlay et al, Appl. Phys. Lett. 95 (2009) 191913.

[2] R Ishikawa et al, Nature Mater. 10 (2010) 278.

[3] H Yu et al, Angew. Chem. Int. Ed. 52 (2013) 5969.

[4] M Matsukawa et al, Nano Lett. 52 (2014) 5969.

[5] The authors acknowledge our collaborators of Dr. H. Yu, Prof. H.S. Zhou in AIST, and Mr. M. Matsukawa, Dr. T. Hisatomi, Dr. Y. Moriya and Prof. K. Domen in Univ. Tokyo. The work was supported by the Funding Program for World-Leading Innovation R\&D on Science and Technology (FIRST Program).
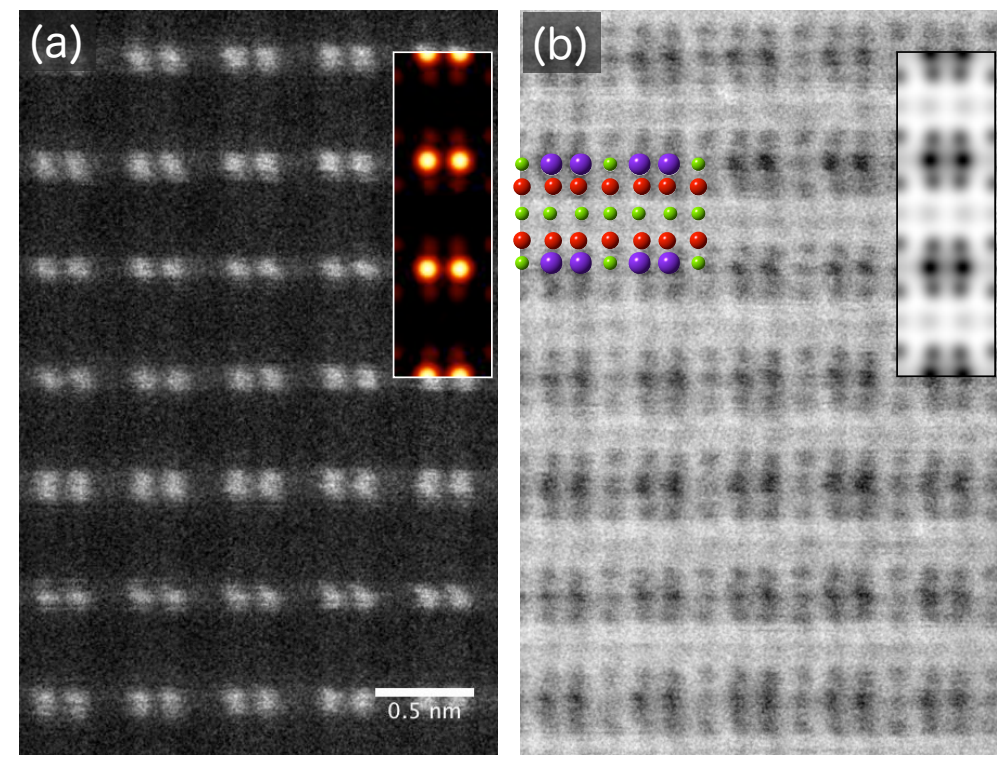

Figure 1. (a), (b) Atomic resolution ADF and ABF STEM images obtained from $\mathrm{Li}_{2} \mathrm{TMO}_{3}$ viewed along the [110] direction, where TM is purple, oxygen is red and lithium is green. The simulated images are overlaid on the right top, respectively.
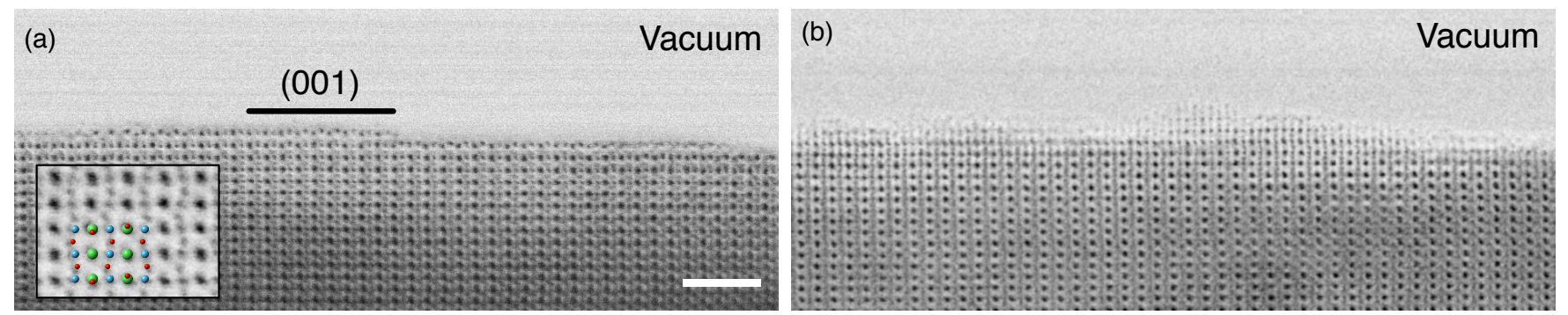

Figure 2. Atomic resolution $\mathrm{ABF}$ STEM images obtained from $\mathrm{LaTiO}_{2} \mathrm{~N}$ viewed along the [100] direction: (a) as-annealed and (b) treated with aqua regia for 15 seconds. The high-magnified image is overlaid on (a). The scale bar is $5 \AA$. 